\title{
Waves and instabilities in an anisotropic universe
}

\author{
D. Papadopoulos ${ }^{1}$, L. Vlahos ${ }^{1}$, and F. P. Esposito ${ }^{2}$ \\ 1 Department of Physics, Aristoteleion University of Thessaloniki, 54006 Thessaloniki, Greece \\ 2 Department of Physics, University of Cincinati, Cincinaty, OH 45219, USA
}

Received 10 July 2001 / Accepted 18 October 2001

\begin{abstract}
The excitation of low frequency plasma waves in an expanding anisotropic cosmological model that contains a magnetic field frozen into the matter and pointing in the longitudinal direction is discussed. Using the exact equations governing finite-amplitude wave propagation in hydromagnetic media within the framework of the general theory of relativity, we show that a spectrum of magnetized sound waves will be excited and form large-scale "damped oscillations" in the expanding universe. The characteristic frequency of the excited waves is slightly shifted away from the sound frequency and the shift depends on the strength of the primordial magnetic field. This magnetic field dependent shift may have an effect on the acoustic peaks of the CMB.
\end{abstract}

Key words. relativity - magnetohydrodynamics (MHD) - waves-cosmology: early Universe

\section{Introduction}

At present not much is known about the existence or absence of magnetic fields in the early Universe. Several scenarios exist for the generation of magnetic fields (see review by Grasso \& Rubinstein 2001). The implications of the primordial magnetic fields for the formation and evolution of the observed structures have been the subject of continuous theoretical investigation.

The observed magnetic field in the clusters and galaxies is in energy equipartition with the gas and the cosmic rays in these systems. The observed magnetic field may be primordial in nature, being left over from the early universe, or created when the first structures formed. Even if we assume the presence of a protogalactic dynamo, to explain the magnitude of the magnetic field still requires a small primordial field. It is a difficult task to determine if the origin of the cluster or the galactic magnetic field is primordial. In contrast, the role of the magnetic field in the fluctuations spectrum of the cosmic microwave background anisotropies can provide bounds in the large-scale magnetic field of the early universe (see Adams et al. 1996; Barrow et al. 1997; Enqvist 1997; Olinto 1997; Durrer et al. 1998).

Many recent studies used a Newtonian or a FriedmannRobertson-Walker (FRW) model for the evolving Universe and super-impose a large-scale ordered magnetic field.

Send offprint requests to: D. Papadopoulos, e-mail: papadop@astro.auth.gr
The magnetic field is assumed to be too weak to destroy the Robertson-Walker isotropy. The anisotropy induced by the magnetic field is treated as perturbation (Durrer et al. 1998; Ruzmakina \& Ruzmakin 1971; Tsagas \& Barrow 1998). Current observations give a strong motivation for the adoption of a FRW model (see reviews above) but the uncertainties on the cosmological standard model are several and the limits of the approximations and the effects one losses by neglecting the anisotropy of the background magnetic field should be investigated.

In this article, departing from the traditional approach, we adopt an anisotropic cosmological model, developed initially by Thorn (1967) and Jacobs (1968). In this model the magnetic field is responsible for the initial anisotropic expansion of the universe. The model is homogeneous and has two equivalent "transverse" directions and one inequivalent "longitudinal" direction at each point in space time. It contains a perfect fluid obeying a "stiff" equation of state $p=\gamma \rho$, with $1 / 3 \leq \gamma \leq 1$. The magnetic field is frozen into the matter and directed along the longitudinal direction.

We analyze the stability of the linear perturbations using the above model. Magnetized density perturbations were studied in detail in Kim (1996) using a Newtonian model for the evolving Universe. Several authors used the FRW model to study magnetized perturbations in the radiation era (see Holcomb \& Tajima 1989), the dissipation of MHD waves in recombination era (Jedamzik et al. 2000) and in the inflanatory era (Brevik \& Sandvik 2000). 
The propagation of the magnetosonic waves in subhorizon and super-horizon scales were discussed by Tsagas \& Maartens (2000a) and more recently a magnetized Biannchi I background model was used to study the coupling of the magnetism with the geometry (Tsagas \& Maartens 2000b).

The main scope of this study is to search for the spectrum of the unstable low frequency plasma waves in an anisotropic model for the universe, using the formalism developed by Papadopoulos \& Esposito (1982). In Sect. 2 we present the basic equations used and in Sect. 3 we perturb the MHD equations keeping first order terms and searching for solutions when the perturbed quantities have the form of a plane wave. We derived the linear dispersion relation for the magnetosonic waves and search for the spectrum of excited waves.

\section{Basic equations}

The general relativistic version of the magnetohydrodynamic equations are:

$$
\begin{aligned}
& \dot{x} u^{a}+x \dot{u}^{a}+x \theta u^{a}+\left(p+\frac{H^{2}}{2}\right)_{; b} g^{a b} \\
& \quad-\left(H^{a} H^{b}\right)_{; b}=0 \\
& \left(\epsilon-\frac{H^{2}}{2}\right)_{; a b} u^{a} u^{b}=h^{a b}\left(p+\frac{H^{2}}{2}\right)_{; a b}+2\left(H^{2} \theta\right) \\
& -\left(H^{a} H^{b}\right)_{; a b}+2 x\left(\frac{2 \theta^{2}}{3}+\sigma^{2}-\omega^{2}-\dot{u}^{a} \dot{u}_{a}\right) \\
& +\frac{x}{2}\left(\rho+3 p+H^{2}\right)+2 \dot{u}_{a}\left(H^{a} H^{b}\right)_{; b} \\
& +\left(H^{2}\right)_{; a} \dot{u}^{a},
\end{aligned}
$$

and

$$
\frac{\mu \dot{H}^{2}}{8 \pi}=\frac{\mu}{4 \pi} \sigma_{i j} H_{i} H_{j}-\frac{4 \theta}{3}\left(\frac{\mu H^{2}}{8 \pi}\right),
$$

where $c=G=1, \mu$ is the permeability, $u^{a}$ is the fluid velocity, $\rho$ is the mass density, $\epsilon=\rho+\rho \Pi, \rho \Pi$ is the internal energy, $\dot{u}^{a}=u_{; c}^{a} u^{c}, \theta=u_{; a}^{a}$ is the expansion velocity, $x=\epsilon+p+H^{2}, H$ the magnetic field and $h^{a b}=g^{a b}+u^{a} u^{b}$. The Eqs. (1), (2), and (3) were perturbed using also the the condition $\delta g_{a b}=0$ known as Cowling approach, and keeping only first order terms in pressure, density, velocity, and magnetic field (see Eqs. (47)-(49) in Papadopoulos \& Esposito 1982).

We linearize the perturbed and search for the amplification or damping of small amplitude hydromagnetic waves in the early universe described by the well known anisotropic cosmological model (Thorn 1967),

$\mathrm{d} s^{2}=-\mathrm{d} t^{2}+A^{2}\left(\mathrm{~d} x^{2}+\mathrm{d} y^{2}\right)+W^{2} \mathrm{~d} z^{2} ;$

here, (i) the model contains a perfect fluid obeying the equation of state $p=\gamma \rho$ where $\frac{1}{3}<\gamma \leq 1$, (ii) the fluid is assumed to be co-moving with the coordinate system and therefore $u^{\mu}=(-1,0,0,0)$ and (iii) as seen in the rest frame of the fluid there is a magnetic field of strength $H$ pointing in the $z$-direction but no electric field. The functions $A$ and $W$ which enter into the line element are $A=A(t)=t^{1 / 2}$ and $W=W(t)=t^{l}$, while

$$
\begin{aligned}
& \rho=\frac{3-\gamma}{16 \pi t^{2}(1+\gamma)} \\
& H=\frac{(1-\gamma)^{1 / 2}(3 \gamma-1)^{1 / 2}}{2 t(1+\gamma)}
\end{aligned}
$$

and $l=(1-\gamma) /(1+\gamma)$.

The ratio of the cyclotron $\left(\omega_{\mathrm{b}}\right)$ to plasma $\left(\omega_{\mathrm{p}}\right)$ frequency is a function of $\gamma\left(\omega_{\mathrm{b}}^{2} / \omega_{\mathrm{p}}^{2}\right)=(2(1-\gamma)(3 \gamma-1)) /(3-$ $\gamma)$. The cyclotron frequency tends to zero as $\gamma \rightarrow 1$ or $\gamma \rightarrow 1 / 3$ and the ratio $0 \leq \omega_{\mathrm{b}}^{2} / \omega_{\mathrm{p}}^{2} \leq 0.3$ remains bounded when $1 / 3 \leq \gamma \leq 1$.

The Standard Hot Big Bang (SHBB) is roughly divided into two regimes, the radiation dominated era, for which the $\gamma=1 / 3$, and the (pressurless) matter dominated era, for which $\gamma \simeq 0$. Thus, at first, it seems not possible for the Universe to experience a stiffer equation of state, with $1 / 3<\gamma \leq 1$. However, with a simple extension of SHBB it is possible to achieve this in a rather natural way.

One prominent example is cosmologies which include a scalar field component in the matter content of the Universe. A homogeneous scalar field $\phi=\phi(t)$ can be treated as an ideal gas with density $\rho_{\phi}=\rho_{\text {kin }}+V$ and pressure $p_{\phi}=\rho_{\text {kin }}-V$, where $\rho_{\text {kin }}=(1 / 2) \dot{\phi}^{2}$ and $V=V(\phi)$ are the field's kinetic and scalar potential energy respectively. In the case $V \gg \rho_{\text {kin }}$, then $\gamma_{\phi} \simeq-1$ and if $\rho_{\phi}$ dominates the Universe then the latter is forced to undergo accelerated expansion. Such a situation, if taken to occur at the very first stages of the Universe evolution, is the typical realization of inflation, in which $\phi$ is called the inflaton field (Olive 1990). At the end of the inflationary period $\phi$ usually oscillates around the minimum of $V(\phi)$ and decays into other particles creating the thermal bath of the SHBB. However, non-oscillatory models of inflation exist in which the inflation does not decay at the end of the inflation but, instead, transforms into a rapid roll-down of its steep potential (Felder et al. 1999). In such models the Universe during this phase continues to be dominated by the scalar field but this time we have $V \ll \rho_{\text {kin }}$ so that $\gamma_{\phi} \simeq 1$. This period is usually referred to as kination or deflation (Joyce \& Procopec 1998). Eventually kination ends and the SHBB begins. At the transition between kination and radiation domination we have a change of $\gamma$ from $1 \rightarrow 1 / 3$.

In the next section we derive the dispersion relation for the low frequency waves, assuming that all the perturbed quantities $\left(\delta \rho, \delta p, \delta H^{\mu}, \delta u^{\mu}\right)$ can be expressed in the form $\mathrm{e}^{i(n t-k z)}$. 


\section{Excitation of low frequency plasma waves}

We assume that the wave vector and the external magnetic field are along the $z$-axis $H^{\mu}=\left(0,0, H^{3}\right)$, $k^{\mu}=(0,0,0, k)$, and the perturbations of the fluid velocity and the magnetic field have a general form $\delta H^{\mu}=$ $\left(\delta H^{1}, \delta H^{2}, \delta H^{3}\right), \quad \delta u^{\mu}=\left(\delta u^{0}, \delta u^{1}, \delta u^{2}, \delta u^{3}\right)$. After some long but straigh forward calculations, we derive the generalized dispersion relation,

$$
\begin{aligned}
-n^{2} & +k^{2} c_{\mathrm{s}}^{2}-\left(1+c_{\mathrm{s}}^{2}\right)\left[-\frac{8}{3} \theta^{2}+2 \sigma^{2}+\frac{1}{2}(\rho+3 p)\right. \\
& \left.+\frac{2 H_{, 0}^{2} \theta}{\rho+p}+\frac{4 \theta^{2} H^{2}}{3(\rho+p)}-\frac{2 H^{2}}{3}\right] \\
& -\frac{1}{2}(p+\rho)\left(1+3 c_{\mathrm{s}}^{2}\right) \\
& -i n\left[4 \theta+\frac{2 H_{, 0}^{2}}{(\rho+p)}-\frac{4 \theta H^{2}}{3(\rho+p)}\right] \\
& =\left(\mathcal{R}_{1}+i n \mathcal{R}_{2}\right) \frac{\delta H^{3}}{\delta \epsilon},
\end{aligned}
$$

and

$\frac{\delta H^{3}}{\delta \epsilon}=\frac{H^{3}}{4 \pi(2-\gamma)}\left[1-\Lambda_{1}-i n \Lambda_{2}\right]$,

where the functions $\mathcal{R}_{1}, \mathcal{R}_{2}, \Lambda_{1}, \Lambda_{2}$ and $\mathcal{D}$ are:

$$
\begin{aligned}
\mathcal{R}_{1}= & {\left[H_{3,00}-4\left(\theta H_{3,0}+\theta{ }_{, 0} H_{3}\right)-2 H_{, 0}^{3} \Gamma_{33}^{0}\right] } \\
& +H_{3}\left[-\frac{4}{3} H^{2}+\frac{4}{3} \theta^{2}+2 \sigma^{2}+2(\rho+2 p)\right] \\
& +g^{33}\left[-2 \Gamma_{33,0}^{0}-2 \Gamma_{33}^{0} \Gamma_{b 0}^{b}+2 \Gamma_{33}^{0} \Gamma_{30}^{3}\right] H_{3} \\
& -\left(n^{2}+k^{2}\right) H_{3}, \\
\mathcal{R}_{2}= & 2 H_{3,0}-4 \theta H_{3}-\Gamma_{33}^{0} H_{3} g^{33}, \\
\Lambda_{1}= & \frac{1}{\mathcal{D}}\left[k^{2}\left(H^{3}\right)^{2}+(2 \gamma-1) \theta\left[2 x \Gamma_{30}^{3}+\left(p+H^{2} / 2\right)_{, 0}\right]\right. \\
& \left.-k^{2} c_{\mathrm{s}}^{2} g^{33}(\epsilon+p)\right], \\
\Lambda_{2}= & (2 \gamma-1) x \theta, \\
\mathcal{D}=- & n^{2} x+k^{2}\left(H^{3}\right)^{2}-(2-\gamma) \theta\left[2 x \Gamma_{30}^{3}+\left(p+\frac{H^{2}}{2}\right)_{, 0}\right] \\
& -i n\left[2 x \Gamma_{30}^{3}+p+\frac{H^{2}}{2}-(2-\gamma) x \theta\right] .
\end{aligned}
$$

In the absence of a magnetic field (e.g. $\gamma=1 / 3$ ) the model used here reduces to a the standard FRW model without a cosmological constant, since $A(t)=W(t)=t^{1 / 2}$ and the real part of the dispersion relation (Eq. (5)) has a very simple form

$$
\begin{aligned}
n^{2}= & k^{2} c_{\mathrm{s}}^{2}+\left(1+c_{\mathrm{s}}^{2}\right)\left[\frac{8}{3} \theta^{2}-\frac{1}{2}(\rho+3 p)\right] \\
& -\frac{1}{2}(p+\rho)\left(1+3 c_{\mathrm{s}}^{2}\right) .
\end{aligned}
$$

Equation (7) reduces further if we use an Einstein cosmological model with cosmological constant $\Lambda=(1 / 2)(\rho+$ $3 p)$, which makes the medium static and homogeneous and $\theta=0$. Using the above assumptions the Jeans instability $\left(n^{2}<0\right)$ for a static isotropic and homogeneous model was derived by in Jackson (1972). The Jeans instability is quenched by expansion $(\theta \neq 0)$ in the FRW model, without a cosmological constant, since the second term in the right hand side of Eq. (7) is always positive and dominates over the third which drives the Jeans instability in the static model.

It is easy to verify that the dispersion relation (Eq. (5)) is simplified considerably in the limit $t \rightarrow 0$,

$$
\begin{aligned}
& -n^{2}\left[1-\frac{u_{A}^{2}}{2-\gamma}\right]+k^{2} c_{\mathrm{s}}^{2}+k^{2} u_{\mathrm{A}}^{2} \frac{1}{2-\gamma} \\
& +T_{1}(\gamma, t)+i n T_{2}(\gamma, t)=0,
\end{aligned}
$$

where $c_{\mathrm{S}}$ is the sound speed and $u_{\mathrm{A}}^{2}=H^{2} / 4 \pi \rho$ is the Alfven speed. The functions $T_{1}$ and $T_{2}$ have the following functional form

$T_{1}(\gamma, t)=\frac{\tilde{T}_{1}(\gamma)}{t^{2}}$

$$
\begin{aligned}
\tilde{T}_{1}(\gamma)= & -\left(9 \gamma^{6}-993 \gamma^{5}+4842 \gamma^{4}-6818 \gamma^{3}+2889 \gamma^{2}\right. \\
& -461 \gamma+788) /\left(6(\gamma-2)(\gamma-3)^{2}(1+\gamma)^{2}\right)
\end{aligned}
$$

and

$$
\begin{aligned}
T_{2}(\gamma, t)= & -4 \theta+\frac{4 \theta}{3}\left(\frac{H^{2}}{4 \pi(\rho+p)}\right)+4 \theta\left(\frac{H^{2}}{4 \pi(2-\gamma)}\right) \\
& -\frac{2 H_{, 0}^{2}}{4 \pi(\rho+p)}-\frac{2 H_{3,0} H_{3}}{4 \pi \rho(2-\gamma)} \\
& +\frac{(1-\gamma)}{(\gamma+1) t}\left(\frac{H^{2}}{4 \pi \rho(2-\gamma)}\right)
\end{aligned}
$$

where $\theta=-2 /((1+\gamma) t)$. The function $T_{2}$ depends on $\gamma$ and $t$ since the magnetic field, the pressure and density are all functions of $\gamma$ and $t$. In other words $T_{2}(\gamma, t)$ has the form

$T_{2}(\gamma, t)=\frac{C(\gamma)}{t}$,

where

$C(\gamma)=\frac{45 \gamma^{4}-36 \gamma^{3}-126 \gamma^{2}+212 \gamma-127}{3(\gamma-3)(\gamma-2)(\gamma+1)^{2}}$.

We can easily show that $T_{1}(\gamma, t)$ is positive and $T_{2}(\gamma, t)$ negative for all allowed values of $1 / 3 \leq \gamma \leq 1$.

We re-write Eq. (8) in the form $D_{\mathrm{r}}+i D_{\mathrm{i}}=0$ and assuming that the the real part of the excited frequency is much larger than the imaginary part, $n_{\mathrm{r}} \gg n_{\mathrm{i}}$, we obtain the frequency of the excited wave from the equation $D_{\mathrm{r}}=0$,

$$
\begin{aligned}
n_{\mathrm{r}}^{2}\left[1-\frac{u_{\mathrm{A}}^{2}}{2-\gamma}\right]= & k^{2} c_{\mathrm{s}}^{2}+\frac{k^{2} u_{\mathrm{A}}^{2}}{2-\gamma} \\
& +\frac{\tilde{T}_{1}(\gamma)}{t^{2}} .
\end{aligned}
$$


and the imaginary frequency from the well-known relation $n_{\mathrm{i}}=-D_{\mathrm{i}}\left(n_{\mathrm{r}}, k\right) /\left(\partial D_{\mathrm{r}}\left(n_{\mathrm{r}}, k\right) / \partial n_{\mathrm{r}}\right) \quad($ see Krall \& Trivielpiece 1973)

$$
\begin{aligned}
n_{i} & =\frac{T_{2}(\gamma, t)}{2\left(1-u_{\mathrm{A}}^{2}(t) /(2-\gamma)\right)} \\
& =\left(\frac{C(\gamma)}{t}\right)\left(\frac{1}{2\left(1-u_{\mathrm{A}}^{2}(t) /(2-\gamma)\right)}\right),
\end{aligned}
$$

were $n_{\mathrm{i}}$ has a large negative value when $t \rightarrow 0$ and decay fast at later times. The role of magnetic field is to make the expanding universe even more stable against the expected Jeans type instabilities discussed early.

The real frequency on the other hand is shifted away from the sound speed

$$
n_{\mathrm{r}}^{2}=\frac{k^{2} c_{\mathrm{s}}^{2}+\frac{k^{2} u_{\mathrm{A}}^{2}}{2-\gamma}+\frac{\tilde{T}_{1}}{t^{2}}}{1-\frac{u_{\mathrm{A}}^{2}(t)}{2-\gamma}}
$$

as $\gamma \rightarrow 1 / 3$ the magnetic field approaches zero and the anisotropic model used here approaches the weaklymagnetized FRW model used extensively in the literature. The characteristic frequency $n_{\mathrm{r}}$ will approach the frequency $n_{\mathrm{r}}=k c_{\mathrm{s}}+\Delta n_{\mathrm{r}}\left(u_{\mathrm{A}}\right)$. The presence of the Alfven speed in the frequency $n_{\mathrm{r}}$ in Eq. (12) may be responsible for the distortion of acoustic peaks, as it was pointed out first by Adams et al. (1996).

The imaginary part is negative $n_{\mathrm{i}}<0$ and a spectrum of low frequencies will be excited with frequency $n_{\mathrm{r}}$. The linear density perturbation will have the form

$\delta \rho \sim\left[\rho_{0} \mathrm{e}^{|B(\gamma, t)|}\right] \mathrm{e}^{i\left(n_{\mathrm{r}} t-k z\right)}$

were $B(\gamma, t)=C(\gamma) /\left(2\left(1-u_{\mathrm{A}}^{2}(t) /(2-\gamma)\right)\right)$. $|B(\gamma, t)|$ is a decaying function of time. For $t \rightarrow 0, \gamma \rightarrow 1$ then $u_{\mathrm{A}} \rightarrow 1$ and $|B(\gamma, t)| \rightarrow \infty$. As $t>>0$ and $\gamma \rightarrow 1 / 3$ then $u_{\mathrm{A}} \rightarrow 0$ and $|B(1 / 3, t)| \rightarrow(3 / 2)$. We then conclude that in the early universe the amplitude becomes large and the excited waves will form a spectrum of damped oscillations in the expanding magnetized universe. We can search for the source of damping by analyzing the functional form of $T_{2}(\gamma, t)$ in Eq. (9). In the absence of a magnetic field $(\gamma=1 / 3) T_{2}=-4 \theta$ and the expanding plasma stabilizes the ion acoustic waves. On the other hand, if the universe is static ( $A=$ constant and $\theta \sim \dot{A} / A \sim 0$ ) the stabilization of the magnetosonic waves is caused by the magnetic pressure. In a magnetized expanding universe (Eq. (9)) both processes will be combined to damp the excited large amplitude oscillations. Assuming, for the sake of argument, that the universe at a certain time $t=$ const. is becoming static, a Jeans type instability sets in. Thus, we can easily realize that the damping is caused by the free energy available both in the plasma and magnetic pressure during the initial stages of its expansion.

\section{Summary}

We analyzed the wave propagation in the early Universe using an anisotropic cosmological model which contains a magnetic field frozen into the perfect fluid with equation of state $p=\gamma \rho$ (with $1 / 3 \leq \gamma \leq 1$ ) and pointing in the $z$-direction. The choice of a stiff equation of state with $1 / 3<\gamma \leq 1$ may be a realistic representation for the evolving universe in the phase between the kination and the radiation era.

The anisotropic model for the early universe used can approach the weakly magnetized FRW model when $\gamma \rightarrow 1 / 3$. In this case the anisotropic model used here approaches the isotropic FRW model were the magnetic field is a small linear perturbation (Durrer et al. 1998; Ruzmakina \& Ruzmakin 1971). Our main results in this article are: (1) The Jeans instability, found early for an Einstein universe with a very particular cosmological constant (Jackson 1972), will be absent since the expansion and the presence of magnetic field will act as a stabilizing force. (2) The anisotropic magnetized Universe re-enforces the stability found initially in the FRW model. (3) A spectrum of low frequency and large amplitude damped oscillations will appear in the early universe with a characteristic frequency $n_{\mathrm{r}}=k c_{\mathrm{s}}+\Delta n$, where $\Delta n$ depends on the strength of the primordial magnetic field. This magnetic field-dependent shift may cause measurable distortion of accoustic peaks (see also Adams et al. 1996; Jedamzik et al. 2000; Koh \& Lee 2000; Durrer et al. 2001). The amplitude of the excited waves is large in the early universe and gradually decays.

We then conclude that if the universe has passed through a strongly magnetized anisotropic phase, before the recombination era started, the waves predicted here will be responsible for the formation of large-scale fluctuations, as it has been shown in 2-D numerical simulations (Brandenburg et al. 1996).

Acknowledgements. We grateful to our colleagues Konstantinos Dimopoulos and Christos Tsagas for many stimulating discussions and for making several comments that considerably improved our article.

\section{References}

Adams, J., Danielsson, U. H., Grasso, D., \& Rubinstein, H. 1996, Phys. Lett. B, 388, 253

Barrow, J. D., Fereira, P. G., \& Silk, J. 1997, Phys. Rev. Lett., 78,3610

Battane, E., Florido, E., \& Jimenez-Vicente, J. 1997, A\&A, 326,13

Brandenburg, A., Enqvist, K., \& Olesen, P. 1996, Phys. Rev. D, 54, 1291

Brevik, I., \& Sandvik, H. B. 2000, Phys. Rev. D, 61, 83505

Cowling, T. G. 1941, MNRAS, 101, 367

Durrer, R., Kahniashvil, T., \& Yates, A. 1998, Phys. Rev. D, 58,123004

Durrer, R., Ferreira, P. G., \& Kahniashvil, T., Phys. Rev. D, 61, 43001

Enqvist, K. 1997, [astro-ph/9707300] 
Felder, G., Kofman, L., \& Linde, A. 1999, Phys. Rev. D, 60, 103505

Grasso, D., \& Rubinstein, H. R. 2001, [astro-ph/0009061]

Holcomb, K. A., \& Tajima, T. 1989, Phys. Rev. D, 40, 3809 Jacobs, K. C. 1968, ApJ, 153, 661

Jackson, J. C. 1972, Proc. Roy. Soc. London, A, 328, 561

Jedamzik, K., Katalinic, V., \& Olinto, A. 1998, Phys. Rev. D, 57,3264

Jedamzik, K., Katalinic, V., \& Olinto, A. 2000, Phys. Rev. Lett., 85, 700

Joyce, M., \& Prokopec, T. 1998, Phys. Rev. D, 57, 6022

Kim, E., Olinto, A. V., \& Rosner, R. 1996, ApJ, 468, 28

Koh, S., \& Lee, C. H. 2000, Phys. Rev. D, 62, 83509

Kolb, E. W., \& Turner, M. S. 1990, The Early Universe, Frontiers in Physics.
Krall, N. A., \& Trivielpiece, A. W. 1973, Principles of plasma physics (McGraw-Hill Co., New York)

Olinto, A. V. 1997, Cosmological magnetic fields, Prossedings of the 3rd RESCEU Symp., ed K., Sato, T., Yanagida, \& T., Shiromizu (Tokyo)

Olive, K. A. 1990, Phys. Rep., 190, 307

Papadopoulos, D., \& Esposito, F. P. 1982, ApJ, 257, 10

Ruzmakina, T. V., \& Ruzmakin, A. A. 1971, Sov. Astron., 14, 963

Thorne, K. S. 1967, ApJ, 148, 51

Tsagas, C. G., \& Barrow, J. D. 1998, Class. Quantum Grav., 15,5223

Tsagas, C. G., \& Maartens, R. 2000a, Phys. Rev. D, 61, 83519

Tsagas, C. G., \& Maartens, R. 2000b, Class. Quantum Grav., 17,2215 